\title{
Tolerability of oral sorafenib in pet dogs with a diagnosis of cancer
}

This article was published in the following Dove Press journal: Veterinary Medicine: Research and Reports

\section{Amanda Foskett \\ Christina Manley \\ Rebecca Naramore \\ Ira K Gordon \\ Bridget M Stewart \\ Chand Khanna}

The Oncology Service, LLC, Leesburg, VA, USA
Correspondence: Chand Khanna Ethos Discovery, 4926 Wisconsin Avenue, Washington, DC 20016, USA

Tel + I 70345 I 8900

Email ckhanna@ethosvet.com

\begin{abstract}
Sorafenib is a multi-target small molecule inhibitor of the RAF kinase family and VEGFR-2/PDGFR. The US Food and Drug Administration approved sorafenib in human patients with liver, thyroid, or renal carcinoma. The aim of this study was to help guide future pharmacokinetic (PK) studies of sorafenib in dogs with a cancer diagnosis. Client-owned dogs were eligible if they had a cytologic or histologic diagnosis of cancer. Patients were enrolled at escalating doses of sorafenib. Patients were evaluable for the study if they received at least one dose of sorafenib and presented 1 week later for a follow-up examination, blood work, and assessment of drug tolerability. The goal of this study was not to define a maximum tolerated dose as may be reasonable in conventional cytotoxic chemotherapy drugs, but rather to describe the tolerability of this drug in dogs with a cancer diagnosis, as a prequel to future sorafenib PK studies. No patients in the study had any evidence of adverse events that were attributable to sorafenib. Doses of $3 \mathrm{mg} / \mathrm{kg}$ were well tolerated and associated with a suggestion of clinical activity, supportive of future PK, and pharmacodynamic analysis. Such future studies are recommended at this dose to define the associated exposure achieved and determine a reasonable schedule for sorafenib administration.
\end{abstract}

Keywords: sorafenib, cancer, tolerability study, developmental therapeutics

\section{Introduction}

Sorafenib is a synthetic compound inhibiting a variety of signal transduction pathways relevant to cancer. ${ }^{1,2}$ Specifically, sorafenib targets RAF/MEK/ERK signaling pathway which is expected to be valuable in cancer due to the important cellular processes regulated by the pathways that are relevant to the cancer phenotype, the so-called hallmarks of cancer, most notably cell growth and survival. ${ }^{3}$ In addition to MAPK inhibition, sorafenib also targets VEGFR-2 and PDGFR. This distinctive spectrum is the basis of its development in human patients. The goal of this study was to define a well-tolerated oral dose of sorafenib in dogs with cancer, as a first step toward its evaluation in dogs. To date, few small molecule inhibitors have been investigated in veterinary oncology. The only Food and Drug Administration (FDA)-approved inhibitor, toceranib phosphate, is structurally similar to the human drug, sunitinib, which is labeled for use in renal cell carcinoma and gastrointestinal (GI) stromal tumor. Toceranib is labeled for the treatment of mast cell tumors, although it is used commonly for the treatment of other tumor types due to known inhibition of multiple targets. These targets include split-kinase family members including VEGFR, PDGFR, Kit, CSF-1, and Flt-3. Unlike sorafenib, toceranib does not target the RAF/MEK/ERK signaling 
pathway, making sorafenib an attractive investigational drug for veterinary patients.

In human cancer patients, sorafenib is FDA approved for the treatment of liver, thyroid, and renal carcinomas. ${ }^{4-6}$ Given the widely recognized shared biological underpinnings of human and canine cancers, it is reasonable to investigate whether this agent has therapeutic activity in canine patients with naturally occurring cancers as defined based on tumor histology, biological behavior, or alterations in similar oncogenic pathways. ${ }^{7-10}$ In addition, a further rationale has received recent support by the identification of a mutation to $B-R A F \mathrm{~V} 600 \mathrm{E}$, in canine bladder and prostatic cancers, known as $B$-RAF V595E. ${ }^{11,12}$ Evidence for downstream pathway alteration in dog bladder cancer cells included proximate pathway dysregulation, including high levels of pMEK, which significantly decreased following exposure to the B-RAF inhibitor, vemurafenib, in canine bladder cancers. ${ }^{11,13}$ Further studies are needed to determine intracellular exposure in vitro, followed by studies to determine if these effects can be safely achieved in vivo.

Preliminary studies in mice, rats, and dogs have shown that sorafenib is well tolerated. In human patients, the most common adverse events include cutaneous skin reactions, diarrhea, and fatigue. ${ }^{14,15}$ In healthy female beagle dogs, mild side effects included diarrhea, reduced body weight, and emesis; however, when doses were reduced to below $10 \mathrm{mg}$ / $\mathrm{kg} /$ day, there were no significant side effects observed. ${ }^{16,29}$

Drug tolerability in purpose-bred research dogs is often distinct from what is observed in pet dogs with a cancer diagnosis. As such, these beagle data are sufficient to propose a starting dose in clinical patients. Pet dogs with cancer are predicted to be more sensitive to similar exposures of the same drugs. Explanations for this increased risk to adverse events include the fact that, compared to purpose-bred research dogs, pet dogs with a cancer diagnosis are often older, affected by the "syndrome" of cancer, and other age-related comorbidities. ${ }^{17,18}$ Accordingly, the goal of this study was to define a single welltolerated once-weekly dose of sorafenib, which could then be used to guide future pharmacokinetic (PK)/pharmacodynamic (PD) studies in dogs with cancer. Such a future study would be used to define an optimal dose/schedule for sorafenib therapy that may be then used to identify "responsive" canine disease indications. After an optimal dose and schedule for sorafenib administration in dogs with naturally occurring cancers is defined, future studies may also explore the clinical value of this kinase inhibitor as a therapeutic for pet animal use and to conduct comparative/translational studies to answer questions related to this drug's use in human patients. Future compara- tive oncology studies may prioritize questions that recognize interindividual and intra-tumoral heterogeneity of naturally occurring dog cancers and to better understand the genomic determinants of the "unexpected" responders. In addition, such studies may be useful to define patterns of resistance and strategies toward selecting the best drug combination to address the expected expansion/emergence of resistant clones.

\section{Patients and methods}

\section{Patient selection}

Client-owned dogs with a cytologic or histologic diagnosis of cancer were eligible. Eligible dogs included any breed, age, sex, and deemed to be healthy with a favorable performance score. Defined as Veterinary Cooperative Oncology Group--Common Terminology Criteria for Adverse Events scores of 0-2 for all systems (grade 0: healthy, grade 1: mild; asymptomatic or mild symptoms; intervention not indicated, grade 2: moderate; minimal, outpatient or noninvasive intervention indication; moderate limitation of activities of daily living) were eligible. ${ }^{19}$

Prior to treatment, a baseline physical examination, complete blood count (CBC), chemistry panel, and urinalysis (UA) were performed. The patients with an absolute neutrophil count of $<2.0 \mathrm{~K} / \mu \mathrm{L}$, platelet count of $<100 \mathrm{~K} / \mu \mathrm{L}$, bilirubin $>2 \times$ upper limit of normal (ULN), and serum creatinine $>2 \times$ ULN were excluded. No criteria related to stage of disease or tumor burden were used to define eligibility in this tolerability study. Patients receiving previous chemotherapy treatment had to have at least a 14-day washout period prior to initiating sorafenib therapy.

The duration a patient was on study was dependent on clinician discretion of the ongoing value and tolerability in a given dog and as such was not preemptively limited or prescribed. The range and mean on the study period for dogs were 7-112 days and 52.5 days, respectively. The study was open to accrual from April 2016 until January 2017.

\section{Sorafenib}

Sorafenib tosylate was obtained from Diamondback Drugs (Scottsdale, AZ, USA). The active pharmaceutical ingredient (API) was validated for identity by the International Analytical Laboratory, in Castle Rock, CO, USA. The active ingredient was assayed using ultrahigh-performance liquid chromatography (UHPLC). This instrument was fitted with a photodiode array having a C18 column and a mobile phase of trifluoroacetic acid and acetonitrile. The reference standard of sorafenib tosylate was obtained from LC Laboratories (Woburn, MA, USA). The identity of the 
sample was confirmed by matching the retention time and ultraviolet (UV) spectrum with that of the reference standard. The tested sorafenib tosylate API powder was determined to be $99 \%$ potent.

\section{Study design}

A prospective, open-label, Phase I, rolling-six, ${ }^{20}$ dose-escalation design was used to assess the tolerability of a single weekly dose of sorafenib. Pet animal patients enrolled in this study were provided care under a clinical trial protocol, inclusive of informed owner consent under the direct care of an institutional veterinarian, following published standards for the conduct of veterinary clinical studies. ${ }^{7,10,21}$ Following owner consent, the first six dogs enrolled in the trial received oral sorafenib at a starting dose cohort of $2 \mathrm{mg} / \mathrm{kg}$. The decision to use this dose was based on the potential for a lengthy half-life that may further accumulate in tumor-bearing dogs. A closely studied dose cohort of normal dogs tolerated $10 \mathrm{mg} / \mathrm{kg}$ daily with minimal GI side effects. ${ }^{16,29}$ Tumor-bearing dogs are more sensitive than young research-bred dogs. We applied the traditional convention of $1 / 3$ of a reasonably tolerated dose with a safety buffer, given that there was an expectation of an extended half-life in tumorbearing dogs. ${ }^{22}$ With planned and implemented dose escalation following our assessment of the starting dose of tolerability, we were able to act in an abundance of caution in these tumorbearing dogs. We used a dose that is approximately $30 \%$ of the $10 \mathrm{mg} / \mathrm{kg}$ dosing used in healthy beagle dogs.

Dogs were observed for any dose-limiting toxicity on a weekly basis with physical exam, $\mathrm{CBC} /$ chemistry, and UA. Adverse events were graded according to VCOG-CTCAE. Adverse events were then assigned as attributable to the drug, the disease, or study participation, using the following modifiers: likely, probable, and unlikely. For the purpose of dose escalation, adverse events were defined as any events $>$ grade 2 (VCOG-CTCAE), likely attributed to drug exposure. As is the convention in most early phase clinical studies in dogs and human beings, there was no control group in this study, which is a known limitation of an early phase clinical trials. Accordingly, adverse event modifiers were included. Such modifiers were assigned to patients based on clinical history, disease, and attending clinician's clinical impression.

Dogs were defined as "evaluable" within a cohort for the purpose of dose escalation, if the intended oral dose was administered and the dog returned for the defined follow-up examination 7 days after dosing for physical examination, $\mathrm{CBC}$, chemistry panel, and UA.

If drug-attributed adverse events were observed in $<2 / 6$ evaluable patients in a cohort, then a subsequent cohort could be accrued, following a 50\% dose escalation. Evaluable patients not experiencing adverse events were permitted to receive additional weekly doses of sorafenib at the discretion of the clinician. A physical examination, CBC, chemistry panel, and UA were performed before any subsequent sorafenib doses. These subsequent doses were not included in the rolling six tolerability assessment; however, all adverse events were evaluated and defined as described earlier. No intra-cohort dose or schedule modification was permitted. The goal of this study was not to determine a maximum tolerated dose (MTD), as is the case for non-cytotoxic drug studies. ${ }^{23}$ Instead, the aim was to determine a well-tolerated dose to guide future PK/PD studies.

\section{Results \\ Patient characteristics}

A total of 13 client-owned dogs were enrolled in the study. A total of 12 patients were deemed evaluable. Six patients were enrolled at an initial dose cohort of $2 \mathrm{mg} / \mathrm{kg}$ given orally, on a once-weekly basis. Six additional patients were then enrolled at a $3 \mathrm{mg} / \mathrm{kg}$ orally on a once-weekly basis. Tumor types included subcutaneous hemangiosarcoma, multi-lobulated osteochondroma, three bladder transitional cell carcinoma (TCC), two oral melanoma, one hepatocellular carcinoma, one metastatic hepatobiliary carcinoma, one presumptive cardiac hemangiosarcoma, and one anal sac apocrine gland adenocarcinoma. Ages ranged from 5 to 14.5 years (Table 1). The majority of patients had received previous chemotherapy

Table I Patient characteristics

\begin{tabular}{ll}
\hline Characteristics & Value \\
\hline $\begin{array}{l}\text { Total number of patients } \\
\text { Gender (\%) }\end{array}$ & I2 \\
$\quad$ Neutered male & $2(16.6)$ \\
$\quad$ Spayed female & $10(83.3)$ \\
Tumor type & 3 \\
TCC & 2 (one subcutaneous and \\
Hemangiosarcoma & one presumed cardiac) \\
& 1 \\
Apocrine gland anal sac adenocarcinoma & I \\
Hepatobiliary carcinoma & $\mathrm{I}$ \\
Hepatocellular carcinoma & $\mathrm{I}$ \\
Renal carcinoma & $\mathrm{I}$ \\
Multilobular osteochondroma & 2 \\
Oral malignant melanoma & \\
Number of prior chemotherapy protocols & \\
0 & 4 \\
I & 5 \\
2 & 2 \\
3 & $\mathrm{I}$ \\
\hline Abbreviation: TCC, transitional cell carcinoma. &
\end{tabular}


protocols prior to enrolling in the trial. Five patients (41\%) had received one prior chemotherapy protocol, two patients $(16 \%)$ had received two prior protocols, and one patient $(8 \%)$ completed three chemotherapy protocols prior to enrollment. Four patients were naive to chemotherapy prior to enrollment in the study.

\section{Adverse events}

One patient in the $3 \mathrm{mg} / \mathrm{kg}$ cohort, with incompletely excised well-differentiated hepatocellular carcinoma, developed significant polyuria and polydipsia (PU/PDip) following the first dose of sorafenib (Table 2). This patient received three additional doses of sorafenib, and the PU/PD was deemed likely attributed to a historical problem with recurrent urinary tract infection and ascending pyelonephritis. One patient in the $3 \mathrm{mg} / \mathrm{kg}$ group developed grade I anemia following a single dose of sorafenib. This anemia was more likely attributable to the underlying disease and tumor bleeding than to drug administration. Two patients experienced one episode of vomiting following the second dose of sorafenib in the $2 \mathrm{mg} / \mathrm{kg}$ cohort. Both of these patients had dietary indiscretion associated with these episodes of vomiting, and, therefore, these events are believed unlikely to be attributable to sorafenib administration. No other patients in this $2 \mathrm{mg} /$ $\mathrm{kg}$ cohort had any adverse events attributed to sorafenib.

\section{Discussion}

This study demonstrated that single oral doses of sorafenib were tolerable up to $3 \mathrm{mg} / \mathrm{kg}$ when given on a once-weekly basis. The decision to conclude the study at this dose was based on the understanding that many targeted molecular agents have a broad therapeutic index without demonstrable adverse events or cytotoxicity. ${ }^{23}$ It is now reasonable to assess the PK exposures associated with such oral doses of sorafenib and to propose a pharmacokinetically appropriate schedule for ongoing dosing with concurrent assessment of relevant PD modulation in cancer cells. The patients in this study represent a diverse population with multiple tumor types. The distribution of age and breed is informative of population of dogs seen in oncology practice. The majority of patients $(66.6 \%)$ had received prior chemotherapy, with an appropriate washout period. This is in line with human Phase I clinical studies, where patients are typically at an advanced stage of disease, refractory to other treatments, and for which there are no other standard therapies..$^{23,24}$ Similarly, veterinary patients enter Phase I clinical trials when there is no standard of care, the patient has become refractory to standard of care, or a clinical trial is more financially feasible than standard of care. ${ }^{22}$

There were no adverse advents noted in this trial which were deemed attributable to sorafenib administration; however, we recognize that without a control group of subjects, it is impossible to say with complete certainty that these adverse events were unrelated to drug administration. This is a limitation of an early-phase clinical study of diseased animals and is not conventional to control for the myriad of factors that may contribute to tolerability of a drug. ${ }^{23,25,26}$ That being said, multiple reasons may explain the lack of adverse events noted in this study. First, this drug formulation administered may not be sufficiently orally bioavailable in the dog. Second, it is possible that the doses used in this study were not high enough to be relevant. Indeed, the starting dose in this study was highly conservative and intended to minimize risk to the patients. Furthermore, the dose escalation could have continued beyond the final dose cohort of this first study if desired. Our selection to start this study with a single $2 \mathrm{mg} /$ $\mathrm{kg}$ once-weekly schedule was based on data suggesting a lengthy half-life for this drug class in research dogs and the potential for drug accumulation to occur in an older population of tumor-bearing dogs, as has been the experience with other small molecule inhibitors in dogs.

Initial sorafenib toxicity studies in healthy research dogs identified the morphological no-effect level after 3 and 12 months to be below $10 \mathrm{mg} / \mathrm{kg} /$ day. Significant toxicities including hematochezia, hair loss, and degenerative processes in the kidneys, hematopoietic system, GI tract, adrenal gland,

Table 2 Adverse events

\begin{tabular}{|c|c|c|c|c|c|c|c|}
\hline & \multirow{2}{*}{$\begin{array}{l}\text { Dosing } \\
\text { group }\end{array}$} & \multirow[t]{2}{*}{ Frequency } & \multirow{2}{*}{$\begin{array}{l}\text { Dose } \\
\text { (mg/kg) }\end{array}$} & \multirow{2}{*}{$\begin{array}{l}\text { Number of } \\
\text { patients }\end{array}$} & \multicolumn{3}{|c|}{ Adverse event } \\
\hline & & & & & Vomiting & Anemia & PU/PD \\
\hline & 1 & Once per week & 2 & 6 & 2 grade $I$ & 0 & 0 \\
\hline & 2 & Once per week & 3 & 6 & 0 & I grade I & 1 \\
\hline Attributable to sorafenib? & & & & & Unlikely & Unlikely & Unlikely \\
\hline
\end{tabular}

Notes: Six patients were enrolled in each dosing cohort. Side effects included vomiting (2), anemia (I), and PU/PD (I). None of these events were deemed attributable to sorafenib administration.

Abbreviation: PU/PD, polyuria/polydipsia. 
teeth, and bone were noted after long-term (3-12 months) once-daily administration of $30 \mathrm{mg} / \mathrm{kg}$ dosing. ${ }^{16,29}$ Accordingly, future studies may safely begin dose escalation at our ending dose cohort. Alternatively, as would be reasonably preferred in the setting of a small molecule inhibitor, we prefer an approach that would more quickly integrate $\mathrm{PK}$ in dogs and PD in dogs or other species to guide both dose and schedules for future sorafenib studies that will assess activity and potentially the features (histology, tumor genomics) of the responding population. Our preference on defining dose/ schedule following PK and PD studies, rather than MTD, is based on the recognition that unlike cytotoxic therapy, it is unlikely that an MTD approach to drug development is optimal..$^{23,24}$ We recognize that our selected approach is lengthy and more greatly prioritizes biological insight. This may be balanced by taking hints from our early data to define optimal study designs and populations for future clinical studies of sorafenib.

Within the constraints of an early phase tolerability study, response to therapy was not a primary end point. Despite this focus, hints of activity may be informative to future investigators and are, as such, briefly summarized in this study. There was a single dog with oral malignant melanoma with a measurable tumor response. This is interesting given the finding in human melanoma patients with NRAS mutations who had a trend toward improved response when treated with sorafenib plus cytotoxic chemotherapy compared to cytotoxic therapy alone. ${ }^{27}$ No other, objective responses were noted in any dogs with measurable disease $(n=10)$. As a result of favorable drug tolerability, all dogs received multiple doses of sorafenib (three to eight doses) on the fixed once-weekly schedule. Of these dogs, no disease progression was noted for greater than 4 weeks in three dogs (all three patients had measurable bladder TCC). Although bladder tumors are notoriously difficult to measure responses, our observation of failure to progress in bladder TCC and a measurable response in melanoma may be "hints" that could guide the future population selection of future early studies of sorafenib in dogs. Although single-agent sorafenib does not appear to have clinical activity in human patients with advanced urothelial cancer, ${ }^{28}$ two recent reports have identified that a large percentage (up to $85 \%$ ) of canine TCC and prostatic tumors carry B-RAF mutations. ${ }^{11,12}$ B-raf mutations have also been reported in canine malignant melanoma, although to a much lesser degree than in human melanoma patients. ${ }^{12}$ To be clear, it is important to limit these "hints" of response to observation only. We realize that patients in this study did not have genetic testing performed on their tumors, and therefore, conclusions cannot be drawn which substantiate these tumors as having a known biological target.

This potential biological activity also supports future PK evaluation of these doses of sorafenib in tumor-bearing dogs. These future studies may combine genetic testing with PK/PD studies to help identify molecular targets in canine patients. These should then be followed by later phase trials to determine activity and efficacy in canine patients with cancer. Due to the emerging uses of sorafenib in human cancer, investigating the optimal use of sorafenib alone and in combination with other anticancer therapies in tumor-bearing dogs may be of value to veterinary and human oncology.

\section{Acknowledgments}

The authors thank the patients, clients, and staff of the Oncology Service, LLC, for helping with this project.

\section{Disclosure}

The authors report no conflicts of interest in this work.

\section{References}

1. Gollob JA, Wilhelm S, Carter C, Kelley SL. Role of Raf kinase in cancer: therapeutic potential of targeting the Raf/MEK/ERK signal transduction pathway. Semin Oncol. 2006;33(4):392-406.

2. Liu L, Cao Y, Chen C, et al. Sorafenib blocks the RAF/MEK/ERK pathway, inhibits tumor angiogenesis, and induces tumor cell apoptosis in hepatocellular carcinoma model PLC/PRF/5. Cancer Res. 2006;66(24):11851-11858.

3. Chang F, Steelman LS, Lee JT, et al. Signal transduction mediated by the Ras/Raf/MEK/ERK pathway from cytokine receptors to transcription factors: potential targeting for therapeutic intervention. Leukemia. 2003;17(7):1263-1293.

4. de Castroneves LA, Negrao MV, de Freitas RM, et al. Sorafenib for the treatment of progressive metastatic medullary thyroid cancer: efficacy and safety analysis. Thyroid. 2016;26(3):414-419.

5. Kane RC, Farrell AT, Madabushi R, et al. Sorafenib for the treatment of unresectable hepatocellular carcinoma. Oncologist. 2009;14(1):95-100.

6. Kane RC, Farrell AT, Saber H, et al. Sorafenib for the treatment of advanced renal cell carcinoma. Clin Cancer Res. 2006;12(24):7271-7278.

7. Khanna C, London C, Vail D, Mazcko C, Hirschfeld S. Guiding the optimal translation of new cancer treatments from canine to human cancer patients. Clin Cancer Res. 2009;15(18):5671-5677.

8. Vail DM, MacEwen EG. Spontaneously occurring tumors of companion animals as models for human cancer. Cancer Invest. 2000; 18(8):781-792.

9. London CA, Hannah AL, Zadovoskaya R, et al. Phase I dose-escalating study of SU11654, a small molecule receptor tyrosine kinase inhibitor, in dogs with spontaneous malignancies. Clin Cancer Res. 2003;9(7): 2755-2768.

10. Paoloni M, Khanna C. Translation of new cancer treatments from pet dogs to humans. Nat Rev Cancer. 2008;8(2):147-156.

11. Decker B, Parker HG, Dhawan D, et al. Homologous mutation to human BRAF V600E is common in naturally occurring canine bladder cancerevidence for a relevant model system and urine-based diagnostic test. Mol Cancer Res. 2015;13(6):993-1002. 
12. Mochizuki H, Kennedy K, Shapiro SG, Breen M. BRAF mutations in canine cancers. PLoS One. 2015;10(6):e0129534.

13. Patrawala S, Puzanov I. Vemurafenib (RG67204, PLX4032): a potent, selective BRAF kinase inhibitor. Future Oncol. 2012;8(5):509-523.

14. Cheng AL, Kang YK, Chen Z, et al. Efficacy and safety of sorafenib in patients in the Asia-Pacific region with advanced hepatocellular carcinoma: a phase III randomised, double-blind, placebo-controlled trial. Lancet Oncol. 2009;10(1):25-34.

15. Lee WJ, Lee JL, Chang SE, et al. Cutaneous adverse effects in patients treated with the multitargeted kinase inhibitors sorafenib and sunitinib. Br J Dermatol. 2009;161(5):1045-1051.

16. Wilhelm S, Chien DS. BAY 43-9006: preclinical data. Curr Pharm Des. 2002;8(25):2255-2257.

17. Kinirons MT, O’Mahony MS. Drug metabolism and ageing. Br J Clin Pharmacol. 2004;57(5):540-544.

18. Roberts DJ, Hall RI. Drug absorption, distribution, metabolism and excretion considerations in critically ill adults. Expert Opin Drug Metab Toxicol. 2013;9(9):1067-1084.

19. Veterinary cooperative oncology group - common terminology criteria for adverse events (VCOG-CTCAE) following chemotherapy or biological antineoplastic therapy in dogs and cats v1.1. Vet Comp Oncol. 2011;14(4):417-446.

20. Skolnik JM, Barrett JS, Jayaraman B, Patel D, Adamson PC. Shortening the timeline of pediatric phase I trials: the rolling six design. J Clin Oncol. 2008;26(2):190-195.

21. Burton J, Khanna C. The role of clinical trials in veterinary oncology. Vet Clin North Am Small Anim Pract. 2014;44(5):977-987.
22. Thamm DH, Vail DM. Veterinary oncology clinical trials: design and implementation. Vet J. 2015;205(2):226-232.

23. Kummar S, Gutierrez M, Doroshow JH, Murgo AJ. Drug development in oncology: classical cytotoxics and molecularly targeted agents. $\mathrm{Br}$ Clin Pharmacol. 2006;62(1):15-26.

24. Dowlati A, Manda S, Gibbons J, Remick SC, Patrick L, Fu P. Multi-institutional phase I trials of anticancer agents. J Clin Oncol. 2008;26(12):1926-1931.

25. Sharyl J, Nass HG. The Role of Clinical Studies for Pets with Naturally Occurring Tumors in Translational Cancer Research: Workshop Summary. Washington, DC: Institute of Medicine Proceedings; 2015.

26. LeBlanc AK, Mazcko CN, Khanna C. Defining the value of a comparative approach to cancer drug development. Clin Cancer Res. 2016;22(9): 2133-2138.

27. Wilson MA, Zhao F, Letrero R, et al. Correlation of somatic mutations and clinical outcome in melanoma patients treated with Carboplatin, Paclitaxel, and sorafenib. Clin Cancer Res. 2014;20(12): 3328-3337.

28. Dreicer R, Li H, Stein M, et al. Phase 2 trial of sorafenib in patients with advanced urothelial cancer: a trial of the Eastern Cooperative Oncology Group. Cancer. 2009;115(18):4090-4095.

29. European Medical Agency [webpage on the Internet]. Initial Marketing Authorization Study of Nexavar. 2007. Available from: http://www. ema.europa.eu/ema/index.jsp?curl=pages/medicines/human/medicines/000690/human_med_000929.jsp\&mid=WC0b01ac058001d124. Accessed March 4, 2017.
Veterinary Medicine: Research and Reports

\section{Publish your work in this journal}

Veterinary Medicine: Research and Reports is an international, peer-reviewed, open access journal publishing original research, case reports, editorials, reviews and commentaries on all areas of veterinary medicine. The manuscript management system is completely online and includes a very quick and fair peer-review system.
Dovepress

Visit http://www.dovepress.com/testimonials.php to read real quotes from published authors. 